\title{
Axon Diameter Mapping in Crossing Fibers with Diffusion MRI
}

\author{
Hui Zhang ${ }^{1}$, Tim B. Dyrby ${ }^{2}$, and Daniel C. Alexander ${ }^{1}$ \\ 1 Microstructure Imaging Group, Department of Computer Science, \\ University College London, London WC1E 6BT, United Kingdom \\ 2 Danish Research Center for Magnetic Resonance, Copenhagen University Hospital, \\ Hvidovre, Denmark
}

\begin{abstract}
This paper proposes a technique for a previously unaddressed problem, namely, mapping axon diameter in crossing fiber regions, using diffusion MRI. Direct measurement of tissue microstructure of this kind using diffusion MRI offers a new class of biomarkers that give more specific information about tissue than measures derived from diffusion tensor imaging. Most existing techniques for axon diameter mapping assume a single axon orientation in the tissue model, which limits their application to only the most coherently oriented brain white matter, such as the corpus callosum, where the single orientation assumption is a reasonable one. However, fiber crossings and other complex configurations are widespread in the brain. In such areas, the existing techniques will fail to provide useful axon diameter indices for any of the individual fiber populations. We propose a novel crossing fiber tissue model to enable axon diameter mapping in voxels with crossing fibers. We show in simulation that the technique can provide robust axon diameter estimates in a two-fiber crossing with the crossing angle as small as $45^{\circ}$. Using ex vivo imaging data, we further demonstrate the feasibility of the technique by establishing reasonable axon diameter indices in the crossing region at the interface of the cingulum and the corpus callosum.
\end{abstract}

\section{Introduction}

Axon diameter mapping using diffusion MRI offers exciting new possibilities for investigating white matter in health and disease beyond diffusion-tensor imaging. Information about axon diameter and density informs the role and performance of white matter pathways 1,2. Specific changes in axon diameter have also been linked to diseases such as multiple sclerosis $[3$ and amyotrophic lateral sclerosis [4. Direct measurement of such features can therefore shed new light into white matter development and disease mechanisms.

Most techniques for axon diameter mapping adopt the model-based strategy in which a geometric model of the tissue predicts the MR signal from water diffusing within. Earlier methods [5,6] assume a single and known orientation of axons in the tissue model, which limits their application to nerve tissue samples or small regions of brain with uniform orientation. More recently, estimating 
axon diameters of unknown orientation on clinical scanners has been shown to be feasible, first in simulation [7], and later in live human brains [8]. However, the models in [7,8 still assume a single albeit unknown fiber orientation. Most recently, Zhang et al 9] relax this assumption to some extent by modeling the axonal-orientation distribution as a Watson distribution. The Watson model accommodates the presence of axonal-orientation dispersion and extends axon diameter mapping beyond the most coherent white matter regions like the corpus callosum to a much wider subset of the white matter. Nevertheless, its model of axonal-orientation distribution remains unimodal. Like the earlier models, it is inappropriate in crossings or partial volume between tracts with significantly different orientations.

Fiber crossings occur in many areas of the brain, resulting in the observation of two or more distinct fiber populations in a significant number of voxels. In such voxels, considerable success has been achieved in resolving the precise underlying crossing fiber configurations. Since the earliest breakthroughs in crossing fiber resolution [10,11, major progress has produced very effective modern techniques (See [12] for a review). Despite that, there has been no attempt at direct measurement of additional microstructure features in these locations. A solution to this problem is prerequisite to realizing whole-brain axon diameter mapping.

This paper describes a technique that addresses the combined problem of crossing fiber resolution and microstructure imaging. We propose a crossing fiber tissue model that enables the simultaneous estimation of crossing configurations and microstructure features. We demonstrate the technique both in simulation and in brain data. The rest of the paper is organized as follows: Section 2 describes the proposed crossing-fiber tissue model and the data fitting procedure; Section 3 details the design of the simulation and brain data experiments for validating the proposed technique and reports the findings; Section 4 summarizes the contribution and discusses future work.

\section{Crossing-Fiber Tissue Model}

The proposed model generalizes the minimal model of white matter diffusion (MMWMD) in [7,8, to accommodate the presence of multiple fiber populations in a single voxel. The MMWMD represents white matter as an ensemble of impermeable cylindrical axons, with both a single diameter and a single orientation, embedded in a homogeneous medium. Water molecules diffuse with an identical intrinsic diffusivity both inside and outside the axons but without exchanges between the compartments. By assuming a single axon diameter rather than a distribution as in 6, 13, the MMWMD enables orientation-invariant estimation of axon diameter for the first time, providing a single summary statistic of the axon diameter distribution, called the axon diameter index [8. The axon diameter index is simpler to estimate than models of the full distribution in [6, 13, but still discriminates naturally occurring axon diameter distributions [8].

To model crossing fibers, we instead represent white matter as a set of $N \geq 1$ distinct fiber populations embedded in a common homogeneous medium. Each 
fiber population is individually modeled as prescribed by the MMWMD. Assuming that no exchange occurs between the fiber populations and their common surrounding medium, as well as between the fiber populations themselves, the normalized (MR) signal from white matter, $A_{w m}$, is thus $\nu_{i c} \sum_{i=1}^{N} f_{i c}^{i} A_{i c}^{i}+(1-$ $\left.\nu_{i c}\right) A_{e c}$, where $\nu_{i c} \in[0,1]$ is the intra-cellular volume fraction within the white matter, $A_{i c}^{i}$ the normalized signal from the $i$-th fiber population with a volume fraction $f_{i c}^{i} \in[0,1]$ relative to the whole population of fibers, and $A_{e c}$ the normalized signal from the extra-cellular medium.

The MMWMD contains two additional compartments: one models isotropically free Gaussian diffusion to capture the partial volume with cerebrospinal fluid (CSF); the other models isotropically restricted diffusion to capture observed restrictions parallel to axons in fixed tissue, potentially due to water trapped within glial cells [5]. Here we include only the latter, because the white matter area considered in our brain data experiment is several voxels away from the boundary with the ventricles and thus void of CSF contamination. The full normalized signal model, $A$, is therefore $\left(1-\nu_{i r}\right) A_{w m}+\nu_{i r} A_{i r}$, where $A_{i r}$ is the normalized signal from the isotropically restricted compartment with a volume fraction $\nu_{i r}$. We set $A_{i r}=1$, following the stationary water assumption in [8], i.e., the compartment signal remains unattenuated by diffusion weighting. The subsequent sections detail the modeling of $A_{i c}^{i}$ and $A_{e c}$ and the fitting procedure.

Intra-Cellular Model. Water diffusion in this compartment is cylindrically restricted. The intra-cellular signal from the $i$-th fiber population, $A_{i c}^{i}$, depends on the axon diameter and orientation of the population, denoted by $a_{i}$ and $\boldsymbol{n}_{i}$ respectively, the intrinsic diffusivity of water, denoted by $d$, as well as the imaging protocol. For the pulsed-gradient spin-echo (PGSE) sequence, as used in our experimental evaluation, we compute $A^{i c}$ using the Gaussian phase distribution approximation [14] as in [7,8] to model the signal from restricted diffusion.

Extra-Cellular Model. Water diffusion in this compartment is hindered. The extra-cellular signal, $A_{e c}$, is modeled with simple (Gaussian) anisotropic diffusion using an (apparent) diffusion tensor as in [15. We model the diffusion tensor, $\mathbb{D}_{h}$, as $\sum_{i=1}^{N} f_{i c}^{i} \mathbb{D}_{c y l}\left(\nu_{i c}, \boldsymbol{n}_{i}, d\right)$, where $\mathbb{D}_{c y l}\left(\nu_{i c}, \boldsymbol{n}_{i}, d\right)$ is the diffusion tensor representing the hindered diffusion in the $i$-th fiber population, defined according to the MMWMD. This follows from the common medium assumption, i.e., water exchanges freely within different regions of the extra-cellular space.

Model Fitting. We fit the proposed model to data with the three-stage routine described in 8]. It provides robust estimates of the model parameters with the Rician Markov Chain Monte Carlo (MCMC) procedure in [7, after an initial grid search and then gradient descent to determine the maximum likelihood (ML) estimates of the parameters.

The full set of model parameters are the $N$ axon diameters $\left(a_{1}, a_{2}, \ldots, a_{N}\right)$ and orientations $\left(\boldsymbol{n}_{1}, \boldsymbol{n}_{2}, \ldots, \boldsymbol{n}_{N}\right)$, the $N-1$ relative volume fractions $\left(f_{i c}^{1}, f_{i c}^{2}\right.$, $\left.\ldots, f_{i c}^{N-1}\right)$, the other two volume fractions $\nu_{i c}$ and $\nu_{i r}$, and the intrinsic diffusivity 
$d$. Note that, because $\sum_{i=1}^{N} f_{i c}^{i}=1$, only $N-1$ of the relative volume fractions are independent. Throughout, we fix $d$ to $0.6 \times 10^{-9} \mathrm{~m}^{2} \mathrm{~s}^{-1}$, its expected value in the ex vivo data, as in [8].

\section{Experiments and Results}

Ex Vivo Imaging of Monkey Data Set. Ex vivo diffusion weighted imaging (DWI) of a 32-month perfusion-fixed Vervet monkey was acquired on a $4.7 \mathrm{~T}$ Varian system with maximum gradient strength $|\boldsymbol{G}|=400 \mathrm{mT} / \mathrm{m}$. (See [16] for brain preparation.) A total of 360 images were collected using a PGSE DWI sequence with single-line spin-echo readout $(\mathrm{TE}=36 \mathrm{~ms}$, $\mathrm{TR}=2500 \mathrm{~ms})$. Each has isotropic $0.5 \times 0.5 \times 0.5 \mathrm{~mm}^{3}$ voxels and 30 sagittal slices centered on the midsagittal plane of the corpus callosum with in-plane matrix size of $128 \times 256$. The protocol, determined using the experimental design optimization [7] adapted for fixed tissue, consists of three HARDI shells with the number of diffusion gradients $[103,106,80],|\boldsymbol{G}|=[300,210,300] \mathrm{mT} / \mathrm{m}, \delta=[5.6,7.0,10.5] \mathrm{ms}$, and $\Delta=$ $[12,20,17] \mathrm{ms}$, corresponding to b-values $=[2084,3084,9550] \mathrm{s} / \mathrm{mm}^{2}$. Ethical rules concerning the handling and care of live animals were followed.

Synthetic Data Experiment. We synthesize diffusion MR signal from a broad set of two-fiber crossing substrates using the publicly available Monte-Carlo diffusion simulator in Camino [17] with the ex vivo imaging protocol described above. Synthetic Rician noise with $\sigma=0.05$ is added to match the SNR of the ex vivo data (around 20). For each substrate, the proposed model with $N=2$ is then fitted to its synthetic data and the parameter estimates are compared against the known ground-truth settings of the substrate.

The synthetic substrates assume reasonable crossing configurations and microstructure features and are constructed as follows: We consider six representative axon diameter combinations: $\left\{a_{1}, a_{2}\right\} \in\{\{2,2\},\{2,4\},\{2,6\},\{4,4\},\{4,6\}$, $\{6,6\}\} \mu \mathrm{m}$. For each combination, we test three relative volume fractions, $f_{i c}^{1} \in$ $\{0.3,0.5,0.7\}$, and a broad range of crossing angles, varying from $30^{\circ}$ to $90^{\circ}$ in $15^{\circ}$ increment. Similar to [7], $\nu_{i c}$ is set to 0.7 , its typical value in brain white matter. Since the diffusion simulator does not model isotropically restricted compartment, $\nu_{i r}$ is 0 . This results in a total of 90 different crossing fiber substrates. Finally, to avoid possible orientation dependence, 20 different instances of each substrate are created by applying random 3-D rotations to the initial configuration. Independent Rician noise described above are added to each instance.

For each substrate, we compute the mean and the standard deviation of the parameter estimates for its 20 random instances. This accounts for the effect of noise and the dependence to orientation. We report our findings from the assessment of all 90 substrates. Due to limit in space, the findings are illustrated with only one axon diameter combination $(\{2,6\} \mu m)$ and $f_{i c}^{1}=0.3$ in Fig. 1 .

The relative volume fraction $f_{i c}^{1}$ can be estimated accurately for all axon diameter combinations and for the crossing angles larger than $45^{\circ}$; for the crossing angle of $30^{\circ}$, it is consistently over-estimated. The intra-cellular volume fraction 


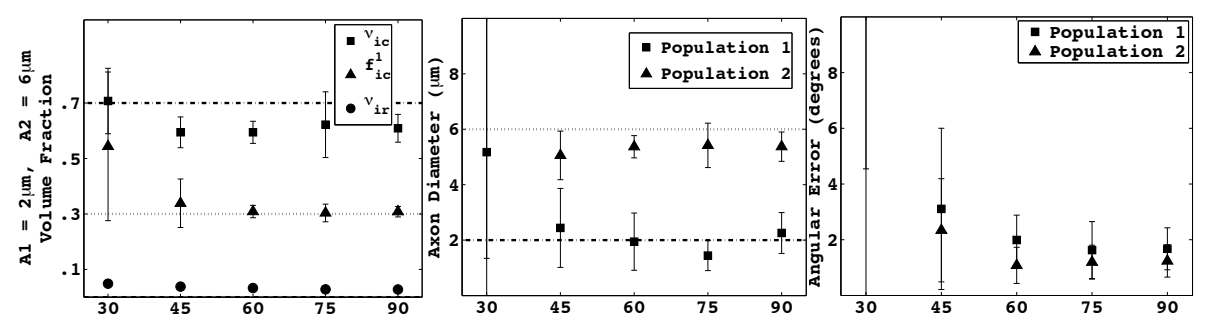

Fig. 1. The parameter estimates for the substrates: $\{2,6\} \mu m, f_{i c}^{1}=0.3$, and all the crossing angles (the horizontal axis)
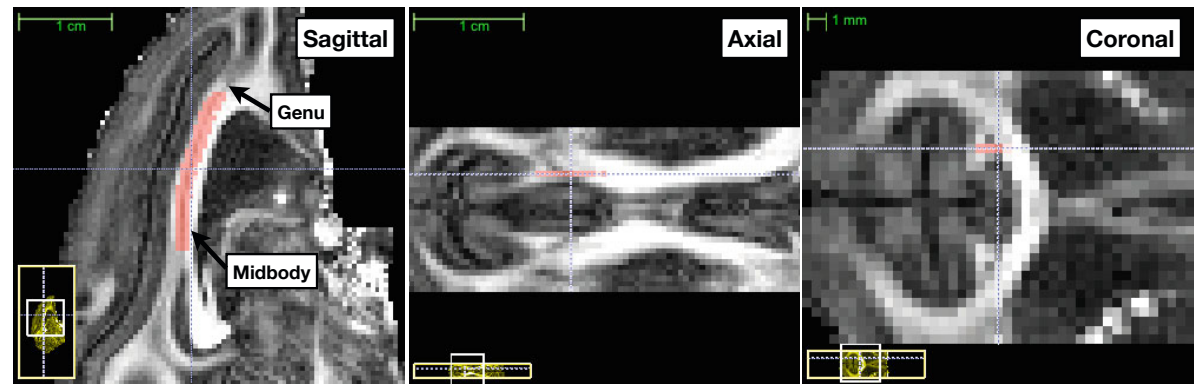

Fig. 2. The manually defined crossing fiber ROI (in red) overlaid on the FA map in three orthogonal views

$\nu_{i c}$ is consistently under-estimated for all substrates, by about $10 \%$. The volume fraction of the isotropically restricted compartment $\nu_{i r}$ can be consistently estimated for all substrates, with a slight over-estimation.

For axon diameters, $2 \mu m$ and $4 \mu m$ are difficult to differentiate from one another for all values of $f_{i c}^{1}$ and crossing angles. However, both can be differentiated from $6 \mu \mathrm{m}$ for all values of $f_{i c}^{1}$ and crossing angles larger than $45^{\circ}$.

Orientations can be estimated accurately for all axon diameter combinations, all values of $f_{i c}^{1}$, and the crossing angles larger than $45^{\circ}$. The error in the orientation estimates increases as the crossing angle decreases and as the axon diameter increases. The error in the estimate of the fiber population with the lower relative volume fraction is higher than that of the other population.

In summary, under practical imaging protocol, the proposed model is able to both resolve the crossing fiber configuration and estimate microstructure features for crossing angles larger than $45^{\circ}$.

Monkey Data Experiment. This experiment uses the ex vivo monkey data set described earlier to demonstrate the efficacy of the proposed model for mapping axon diameters in brain tissue with crossing fibers. We manually delineate a welldefined two-fiber crossing region-of-interest (ROI) between the corpus callosum and the cingulum bundle as shown in Fig. 2. Note the dark band in the center 


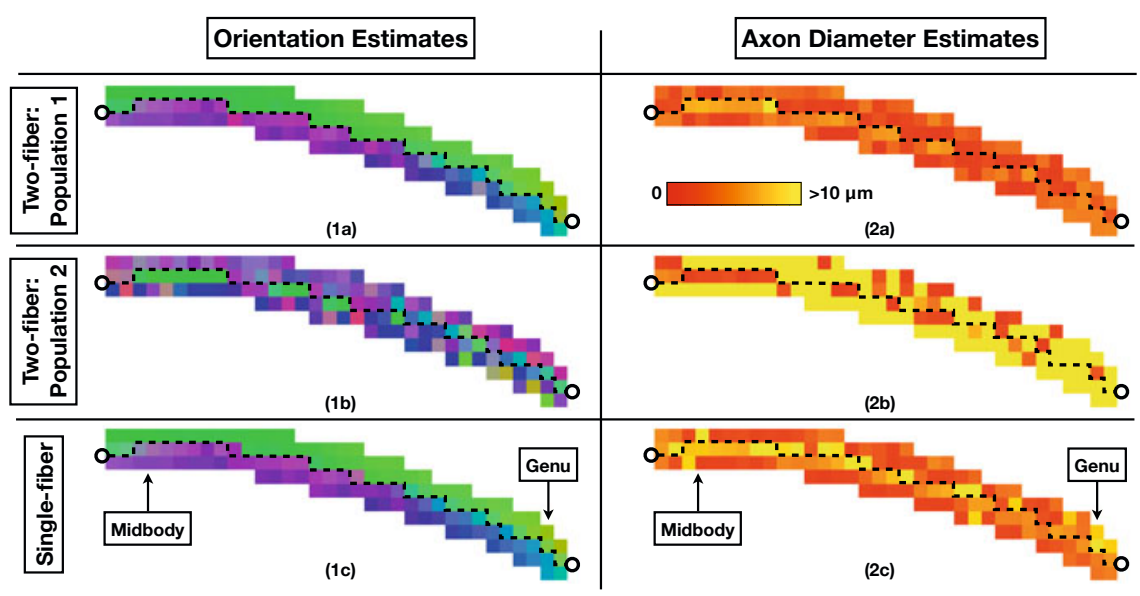

Fig. 3. The orientation and axon diameter estimates for both the two-fiber and single-fiber models. The orientation estimates are represented using the standard RGBencoding (Red: medial-lateral, Blue: inferior-superior, Green: anterior-posterior) [18].

of the ROI. The appearance of such voxels flanked by the ones with higher FA is characteristic of crossing fibers due to partial volume between two tracts with substantially different orientations. We fit both the proposed two-fiber model and the MMWMD (single-fiber) to each voxel in this region, and compare their respective estimates.

Fig. 3 shows the orientation and axon diameter estimates for both the twofiber and single-fiber models. For the two-fiber model, the fiber population 1 corresponds to the population initialized, during model fitting, with the primary eigenvector of the diffusion tensor estimate at each voxel. The well-defined trajectory of the cingulum, which traverses from anterior to posterior, allows us to identify the voxels colored in green in Fig. 3(1a) as the ones with the fiber population 1 being the cingulum. The dashed line separates these voxels from the ones below them, which are primarily the corpus callosum fibers.

The most striking observation is that, despite the orientation estimates of the single-fiber model (Fig. 3(1c)) being largely consistent with those of the fiber population 1 of the two-fiber model (Fig. 3(1a)), their respective axon diameter estimates are distinctly different. In particular, among the voxels immediately adjacent to the dashed line, the putative interface between the cingulum and the corpus callosum, the axon diameter estimates of many, from the single-fiber model (Fig. 3(2c)), are close to or higher than $10 \mu m$, much

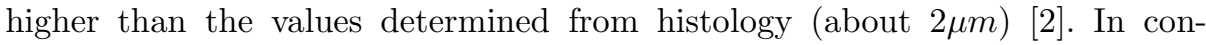
trast, their corresponding estimates for the fiber population 1 from the two-fiber model (Fig. 3(2a)) are in the same range as the histologically estimated values, highlighting a key benefit of using the two-fiber model in such crossing fiber voxels. 
The orientation and axon diameter estimates for the fiber population 2 from the two-fiber model require more care in their interpretation. The most apparent feature of the axon diameter estimates (Fig. 31(2b)) is that a large majority of them are higher than $10 \mu \mathrm{m}$. Further inspection confirms that the values for almost all these voxels are close or equal to $40 \mu \mathrm{m}$, the maximum value allowed in our fitting routine to indicate the negligible presence of the corresponding fiber population, in this case, the fiber population 2. On the other hand, a number of voxels have axon diameter estimates with values comparable to those from histology. They can be divided into two groups according to their spatial locations and orientations. In the first group are the voxels that are immediately adjacent to the putative cingulum and corpus callosum interface and have their orientation estimates for the fiber population 2 consistent with being part of the cingulum, i.e., colored in green in Fig. 33(1b). For these voxels, the two-fiber model is able to both resolve the crossing configuration and estimate the axon diameters of individual fiber populations. In the second group are the voxels that are away from the interface and inside the cingulum region. The fiber population 2 of these voxels may correspond to the outward-projecting cingulum fibers.

\section{Discussion}

We have described a technique for joint estimation of crossing fiber configuration and microstructure features using a new crossing-fiber white matter model that includes individual axon diameter and volume fraction parameters for each fiber population. The results from simulation and brain data demonstrate promising possibilities for extending axon diameter mapping to the whole brain. In particular, the brain data findings suggest that the crossing-fiber model can resolve crossing configurations and estimate axon diameters simultaneously under some circumstances; when it fails to do so, it can still provide more sensible axon diameter estimates for the dominant fiber population. Given that it is challenging to estimate axon diameter in voxels with only a single orientation, it is remarkable that we can make progress at crossing. Even improving the estimate of the dominant popualtion is itself useful and a significant step forward.

Nevertheless, the proposed crossing fiber model can be extended in several ways: First, the current model assumes that axons are strictly parallel within each fiber population. This assumption can be relaxed to account for axon spreading in each distinct population using the orientation dispersion model in [9. Second, the current model assumes that the extra-cellular space is a common homegeneous medium. A more general model may require the modeling of the extra-cellular space of each fiber population individually. Lastly, we will examine other crossing regions, such as the pons and the crossing regions between the corpus callosum and the corona radiata in centrum semiovale.

Acknowledgement. We thank Prof Maurice Ptito, University of Montreal, for providing the monkey brain. The future and emerging technologies program of the EU FP7 framework funds the CONNECT consortium (brain-connect.eu), which supports this work. EPSRC fund DCA under grant EP/E007748. 


\section{References}

1. Ritchie, J.M.: On the relation between fibre diameter and conduction velocity in myelinated nerve fibres. Proc. R. Soc. Lond. B 217, 29-35 (1982)

2. Lamantia, A.S., Rakic, P.: Cytological and quantitative characteristics of four cerebral commissures in the rhesus monkey. J. Comp. Neurol. 291, 520-537 (1990)

3. Shintaku, M., Hirano, A., Llena, J.F.: Increased diameter of demyelinated axons in chronic multiple sclerosis of the spinal cord. Neuropathol. Appl. Neurobiol. 14, 505-510 (1988)

4. Sasaki, S., Maruyama, S.: Increase in diameter of the axonal initial segment is an early change in amyotrophic lateral sclerosis. J. Neurological. Sci. 110, 114-120 (1992)

5. Stanisz, G.J., Szafer, A., Wright, G.A., Henkelman, M.: An analytical model of restricted diffusion in bovine optic nerve. Magn. Reson Med. 37, 103-111 (1997)

6. Assaf, Y., Blumenfeld-Katzir, T., Yovel, Y., Basser, P.J.: AxCaliber: a method for measuring axon diameter distribution from diffusion MRI. Magn. Reson Med. 59, 1347-1354 (2008)

7. Alexander, D.C.: A general framework for experiment design in diffusion MRI and its application in measuring direct tissue-microstructure features. Magn. Reson Med. 60, 439-448 (2008)

8. Alexander, D.C., Hubbard, P.L., Hall, M.G., Moore, E.A., Ptito, M., Parker, G.J.M., Dyrby, T.B.: Orientationally invariant indices of axon diameter and density from diffusion MRI. NeuroImage, 1374-1389 (2010)

9. Zhang, H., Hubbard, P., Parker, G.J.M., Alexander, D.C.: Axon diameter mapping in the presence of orientation dispersion with diffusion MRI. NeuroImage 56, 13011315 (2011)

10. Tuch, D.S., Reese, T.G., Wiegell, M.R., Makris, N., Belliveau, J.W., Wedeen, V.J.: High angular resolution diffusion imaging reveals intravoxel white matter fiber heterogeneity. Magn. Reson Med. 48, 577-582 (2002)

11. Jansons, K.M., Alexander, D.C.: Persistent angular structure: new insights from diffusion magnetic resonance imaging data. Inverse Problems 19, 1031-1046 (2003)

12. Seunarine, K.K., Alexander, D.C.: Multiple fibres: beyond the diffusion tensor. In: Behrens, T.E.B., Johansen-Berg, H. (eds.) Diffusion MRI, pp. 55-72. Elsevier, Amsterdam (2009)

13. Barazany, D., Basser, P.J., Assaf, Y.: In-vivo measurement of the axon diameter distribution in the corpus callosum of a rat brain. Brain 132, 1210-1220 (2009)

14. Murday, J.S., Cotts, R.M.: Self-diffusion coefficient of liquid lithium. J. Chem. Phys. 48, 4938-4945 (1968)

15. Assaf, Y., Freidlin, R.Z., Rhode, G.K., Basser, P.J.: New modeling and experimental framework to characterize hindered and restricted water diffusion in brain white matter. Magn. Reson Med. 52, 965-978 (2004)

16. Dyrby, T.B., Baaré, W.F.C., Alexander, D.C., Jelsing, J., Garde, E., Søgaard, L.V.: An ex vivo imaging pipeline for producing high-quality and high-resolution diffusion-weighted imaging datasets. Hum. Brain Mapp. 32, 544-563 (2010)

17. Hall, M.G., Alexander, D.C.: Convergence and parameter choice for Monte-Carlo simulations of diffusion MRI. IEEE Trans. Med. Imaging 28, 1354-1364 (2009)

18. Pajevic, S., Pierpaoli, C.: Color schemes to represent the orientation of anisotropic tissues from diffusion tensor data: application to white matter fiber tract mapping in the human brain. Magn. Reson Med. 42, 526-540 (1999) 